\title{
Wikis in the Workplace: \\ How Wikis Can Help Manage Knowledge in Library \\ Reference Services
}

Angela Kille

Graduate Student

School of Information

The University of Texas at Austin

\begin{abstract}
This article explores how wikis can be used in library reference services to manage knowledge and why they should be used in this environment. The article begins with a description of wikis, then covers knowledge management and the systems that support knowledge management, specifically collaborative and conversational technologies. Next, the author discusses how wikis can be used as a knowledge management system and explores the organizational applications. Finally, a discussion follows on how wikis can be used to support knowledge management in library reference services with some examples of wikis as both private and public knowledge repositories and as collaborative workspaces.
\end{abstract}




\section{Introduction}

Named after the Hawaiian term for "quick," wikis are interactive Web sites to which users can contribute. In a wiki, any user with a Web browser can add entirely new pages or add new content to existing pages, as well as change or delete existing information. Users can also edit the organization of the wiki, in addition to the content, and need not know HTML or have Web design skills since Web-based forms provide the simple editing interface. The software that runs a wiki is called a wiki engine and there are many free open source wiki engines available to run wikis (e.g., MediaWiki), as well as online wiki providers who will host a wiki for you, (e.g., Seedwiki). Although some wikis are intended for the public, like Wikipedia (http://www.wikipedia.org) or Library Success: A Best Practices Wiki (http://www.libsuccess.org), this technology is now being adopted by organizations as a collaborative tool for managing knowledge among their employees. A wiki can be used in multiple ways as a conversational knowledge management system to support the goals of many organizations, including libraries. This paper explores how wikis can be used in library reference services to manage knowledge and why they are well-suited to this environment.

\section{Wikis}

\section{Definition}

Ward Cunningham created the first wiki in 1995 when he wanted a quick way to publish information collaboratively on the Web (Leuf \& Cunningham, 2001). The original WikiWikiWeb site (http://c2.com/cgi/wiki) created by Cunningham still exists and is actively maintained by users. Cunningham called the wiki "the simplest online database that could possibly work" (2001, p. 15).

Although wiki software can vary, generally most wikis include these simple basic features: (a) wikis are open to all users, or certain authorized users, and can be freely written or edited by those users so Web pages are authored collectively; 
(b) new pages can be added to a wiki by typing a WikiWord, a word containing two or more capital letters, or by other conventions, such as double brackets; (c) wikis use a simplified markup system, not HTML (Hyper Text Markup Language), although some wikis now have WYSIWYG (What You See Is What You Get) editing. Above all, a wiki is a collaborative space and a way to organize and cross-link knowledge (Leuf \& Cunningham, 2001).

Creating and Editing Content

Using a wiki is simple and straightforward. Updates to a page can be made by clicking on the "Edit" or "Edit this page" link on the page. A user can then make the needed changes in plain text and click "Save" or "Update." Some wiki software programs have WYSIWYG editing toolbars with buttons to make formatting changes (e.g., bold, italics, hyperlinks, etc.). Most people will be comfortable with this interface since it is so familiar to anyone who uses word processing programs. Other wiki programs require users to format text in a specific way with punctuation marks around the text to be formatted. For example, typing two asterisks around a word (e.g., ${ }^{* *}$ wiki ${ }^{* *}$ ) makes the word appear bold in some wiki formatting conventions. According to Fichter, "the formatting rules vary based on the type of wiki software and options turned on by the administrator" (2005b, p. 48). The key to this wiki formatting is that it is easy to learn and use. Some wikis also allow HTML formatting as another option for users, but it is not required.

Users can easily create new Web pages in wikis to add content. Fichter states that "many wikis use two or more capitalized words run together to make a wikiname" (2005b, p.48). By typing a wikiname, the user automatically creates a link to a new page. For example, to add a new page about the wiki, the user could type "AboutThisSite." This wikiname syntax automatically creates a link to the new page on the current page being edited and when the user clicks on this link, the user is automatically sent to the editor for the new AboutThisSite page. Some wikis use square brackets around a word to create a new page (e.g., [about] to create a new "about" page). Links to new pages will appear differently than links to existing 
pages. Some wiki programs use a "?" next to the new page link, others use a dotted line beneath the link (instead of solid) to denote that it is a new page with no content. Users can also easily link to external Web sites since wikis recognize URLs and will automatically create hyperlinks when a complete Web address is typed on a wiki page. Adding new images to a wiki page is equally simple. The user typically encloses the URL to the picture in square brackets. 


\section{Organization and Navigation}

Due to its fluid and open structure, a wiki can quickly become disorganized. To address this potential problem, users of a new wiki usually develop or adopt norms and conventions for naming pages and adding content to help the wiki stay consistent (Fichter, 2005b). Fichter also states that "in order to work well, wikis need constant care and pruning" (p. 48). So, some of a wiki's users typically volunteer to spend timing cleaning up the wiki to help maintain the wiki's usefulness. According to Fichter, people who perform this role are called "wiki gardeners" (p.48).

When navigating a wiki, the user must first realize that the standard menu structure found on most Web sites won't be available in a wiki. For example, most wikis have pages set up to orient new users (e.g., NewVisitor or StartingPoints). Some wikis also have RoadMap pages where people "find, collect, and link to useful wiki pages on a particular topic" (Fichter, 2005b, p. 48). In addition, wikis have a RecentChanges page that automatically updates each time a page in the wiki is modified. This page helps users track any changes or discussion taking place in the wiki. Users can also typically receive the recent changes via a RSS (Really Simple Syndication) feed. Some wikis also have a backlink feature that allows the user to see all of the wiki pages that link to the current page. Finally, users can navigate wikis by doing keyword searches to find information.

\section{Wiki Technology}

The wiki software that runs a wiki is frequently called a wiki engine. Wiki engines are usually installed on a server, so running a wiki engine requires more advanced computer skills than those of basic computer users (Clyde, 2005). Most wiki engines are open source and are written in a variety of programming languages. According to Clyde, "one of the advantages of using a wiki engine on one's own server is that the wiki software can be reconfigured or even recoded to meet local needs" (p. 54). Some popular wiki engines include MediaWiki 
(http://www.mediawiki.org) (see Figure 1), PmWiki (http://www.pmwiki.org), MoinMoinWiki (http://moinmoin.wikiwikiweb.de), and TWiki (http://twiki.org).

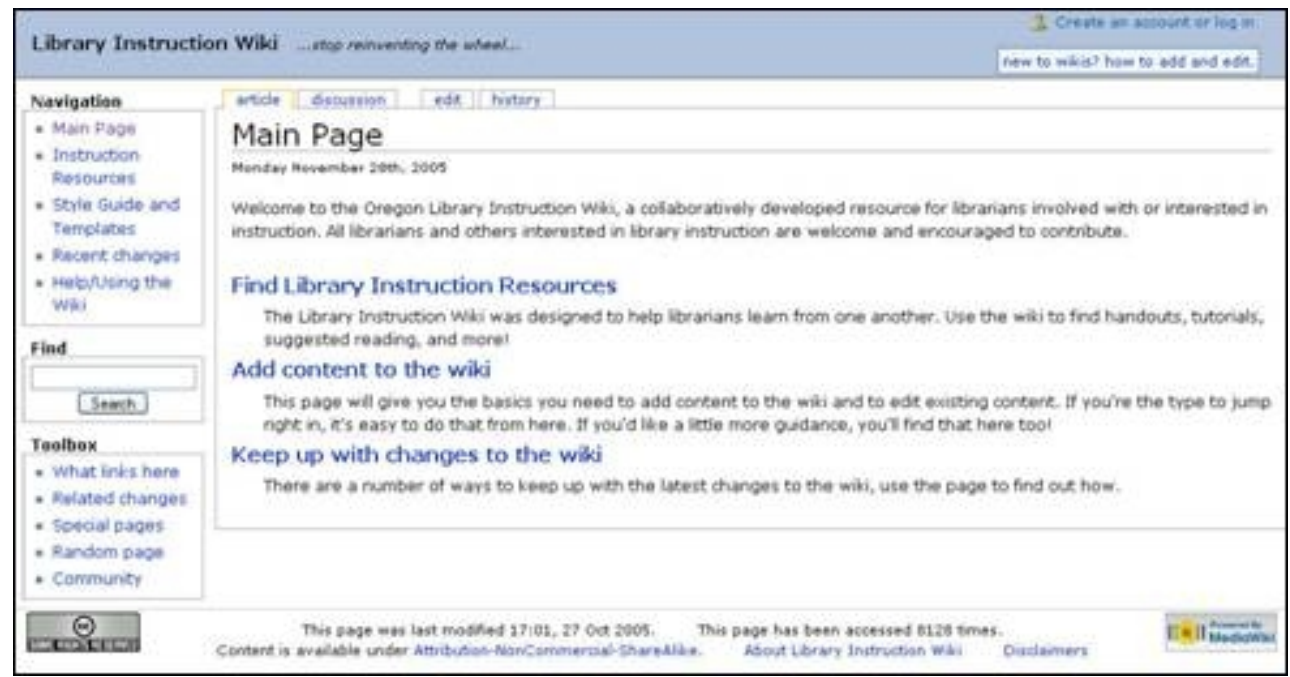

Figure 1. Example of MediaWiki software in use at http://instructionwiki.org. Tonkin (2005) includes two comparison charts of wiki software features. Another comparison table can be found in Wikipedia ("Comparison," 2005). The WikiMatrix Web site (http://www.wikimatrix.org) also features an interactive wiki feature comparison chart.

People interested in using a wiki who do not have the skills or desire to set up their own server can choose from a variety of hosted wikis, or wiki farms. Wiki farms run a wiki engine on their own servers as a service. This option works well for people interested in testing out the wiki concept or for people who don't want to manage a wiki on their own server. Some wiki farms include Seedwiki (http://www.seedwiki.com), PBWiki (http://www.pbwiki.com), XWiki (http://www.xwiki.com), JotSpot (http://www.jot.com), and Socialtext (http://www.socialtext.com). JotSpot is unique since it includes a variety of readyto-use application templates that can be installed. Some examples include an event calendar, company directory, call log application, blog application, and polling application, among many others. Socialtext is a commercial product aimed at businesses and organizations. Some of these hosted wikis have free versions; all have various fee-based services from which to select. 
Before implementing a wiki in the work environment, potential users must first consider the workplace culture and environment, and the potential users and their requirements (Fichter 2005a). According to Fichter (2005b), "wikis work best in organizational cultures in which there is a high level of trust and control can be delegated to the users of the system" (p. 49). Fichter (2005a) also states that you must first define your requirements by considering these issues: communication, sharing, and management. For example, you need to think about how frequently people need to communicate, what technology people prefer, how often groups want to share information, how many potential users would be involved, what organizational or industry standards are in place, and possible training needs. In addition, you must make sure wikis offer the functionality you need and that other collaborative tools are not right for the task (e.g., blogs or discussion forums).

\section{Knowledge Management}

\section{Knowledge}

So, what is knowledge? Data, information, and knowledge have an integrated relationship on a progressive continuum. According to Davenport and Prusak, "data is a set of discrete, objective facts about events" (2000, p. 2). It is the raw material that people use to generate information. Examples of data include lists of names and addresses, raw statistics, or lists of items. Davenport and Prusak identify information as a message, or a form of communication, but information can also be seen as data organized for a specific purpose. Unlike data, information is placed in a meaningful context. So, examples of information could include data about the number of people who were injured in car accidents in Texas last year, a list of all of the materials acquired by a library in the last month, or a list of the resources used to answer a specific customer service question.

Knowledge, however, is much more complex than data or information. Davenport and Prusak define knowledge as:

a fluid mix of framed experience, values, contextual information, and expert insight that provides a framework for evaluating and incorporating new experiences and information. It originates and is applied in the minds of 
knowers. In organizations, it often becomes embedded not only in documents or repositories but also in organizational routines, processes, practices, and norms. (2000, p. 5)

People turn information into knowledge by making comparisons, thinking about potential consequences, making connections, and discussing the information with other people (Davenport \& Prusak, 2000). Knowledge is developed through experience over time and can help people deal with complex issues and judge new situations. Gandhi states, "when information is analyzed, processed, and placed in context, it becomes knowledge" (2004, p. 369). There are also two types of knowledge: tacit and explicit. Tacit knowledge is personal knowledge that is often difficult to formalize or articulate. According to Stover, it is intuitive and practicebased, therefore making it difficult to pass on to others (2004). Some examples of tacit knowledge include hands-on skills, best practices, and special know-how. Articulating tacit knowledge is important for any organization so it does not lose that knowledge if the individual who owns it leaves the organization. In contrast to tacit knowledge, explicit knowledge is easily documented and shared. Examples of explicit knowledge include department routines, standard operating procedures, and organization policies. 


\section{Knowledge Management}

Knowledge management has grown out of the recognition of the value found in knowledge components such as judgment, innovation, and humor. Prusak states that as access to information expands, the value of these components becomes greater (2001). So, knowledge management provides an integrated approach to identifying, managing, and sharing all of an organization's knowledge assets, which includes the expertise and experience of individual employees, with the ultimate goal of helping an organization attain its mission and goals. According to Gandhi, "the goal of KM [knowledge management] is to create a learning and sharing organization by linking together and creating a flow between the buckets of information generated by people in different parts of the company" (2004, p. 368). With knowledge management processes in place, organizations can improve productivity, enhance collaboration, encourage innovation, facilitate the flow of knowledge, promote knowledge sharing, capture and record employee knowledge, and improve customer service (Gandhi, 2004). In addition, with successful implementation of knowledge management initiatives, organizations can more easily learn and adapt to a changing environment.

Knowledge Management and Libraries

Knowledge management practices offer many benefits for libraries. They can help improve efficiency, accuracy, and consistency of information use within the organization. In this way, librarians and administrators can carry out their work more effectively. This management strategy can also facilitate improved communication between all levels of the organization. In addition, knowledge management can help libraries remain competitive in an era of constant change by facilitating the rapid transfer of knowledge. Most importantly, exploiting knowledge can be a major "advantage for libraries, which can translate into better service to users" (Jantz, 2001). Overall, knowledge management can provide a systematic approach to organizing the knowledge of a library's employees, and can make this 
knowledge available to other librarians and staff in order to improve the organization (Jantz).

\section{Knowledge Management Systems}

\section{Definition}

Knowledge management uses technology to provide the formal structure where the knowledge and experience of employees can be systematically captured and shared. These technological structures, or knowledge management systems, facilitate improved access to and transfer of knowledge. Some examples of knowledge management systems include intranets (internal Web sites), groupware applications (software that enables users to share information), e-mail lists, and knowledge mapping tools (representational maps that detail staff expertise and knowledge).

\section{Groupware}

Groupware "is a collaborative technology which allows people to communicate with each other, co-operate on projects and share information and knowledge" (Gunnlaugsdottir, 2003, p. 371). According to this author, groupware links employees together through the groupware application and provides access to needed information while eliminating duplication of effort. So, groupware offers "effective collection, storing, organisation, retrieval and distribution of information" (Gunnlaugsdottir, 2003, p. 372).

Conversational Knowledge Management Systems

Wagner (2005) identifies conversational knowledge management systems as a type of groupware. These conversational technologies include wikis, blogs, and discussion forums. According to Wagner, conversational knowledge management yields "benefits at numerous stages of the knowledge management process beginning with knowledge creation and ending with knowledge use and refinement" (2005, ii). Typically these systems capture and represent conversations while accommodating contextualization, search, and community (Wagner, 2005). Other characteristics include the ability to represent information in 
plain text and build relationships between content in a knowledge repository. Finally, these systems support collaboration between people at different times and locations and are Web based.

Conversational technologies "succeed by harnessing communal knowledge and social capital of groups by supporting the natural process of conversation and documenting its results" (Wagner, 2005, p. vii). These conversational technologies don't require a large amount of time to figure out or a high level of technical skill. Wikis, blogs, and discussion forums are lightweight applications and relatively inexpensive, so many types of organizations could implement them with only moderate resources. These tools all support open and informal communities, but may need additional capabilities to support closed and formal ones (Wagner, 2005). Integration with existing data and tools would also be needed for adoption in an organization. Finally, Wagner (2005) stresses the need to match the organizational culture with the knowledge management technology. Conversational technologies "invite critique, present multiple points of view, and seek to change others' ideas" (Wagner, 2005, p. vii), so organizations must value this type of exchange for the tool to be successful.

Comparison of Conversational Technologies

Wagner (2005) includes a comparison chart of wikis, blogs, and discussion forums (or boards) in his article that summarizes each technology's characteristics and benefits. This chart also takes into account different types of communities and their unique requirements. The most significant differences between these three types of conversational technologies can be found in the mode of conversation, content indexing, and medium (Wagner, 2005). Discussion forums and wikis can both be considered many-to-many communication tools, while blogs are typically used as one-to-many broadcasting tools which can be more difficult to sustain since all the content is created by one individual. Blogs and discussion forums are both primarily indexed by time. This makes these tools useful for news distribution, but not as useful for topics that would be referenced for some time in the future. 
Wagner (2005) states that knowledge management favors topic-based indexing, which is how wikis are organized. Some blogs and discussion forums include topic-based indexing, but usually as a secondary organization method (Wagner, 2005). In addition, wikis have an editing functionality so the most current and refined form of a topic's content is always displayed. Wikis and blogs are completely Web based, while discussion forums have a history of being used with e-mail and mailing lists, although they now are frequently found on the Web. According to Wagner (2005), of these three conversational technologies, wikis appear to be the most versatile for knowledge management applications, although each tool has its beneficial uses.

\section{Wikis as Knowledge Management Tools}

\section{Wikis and Knowledge Management}

Since wikis are a conversational technology, they are most effective when used for ad hoc problems with decentralized knowledge sources (Wagner, 2004). Wikis encourage incremental knowledge creation, so when a page is created, the content can be partial or incorrect since additional collaborators will edit and add more information to the page. In addition, the ability to create hyperlinks to non-existing pages also illustrates the incremental way knowledge is created in a wiki (Wagner, 2004). Wikis also "create joint ownership of the work product" (Wagner, 2004, p. 277). Everyone can contribute and freely share knowledge. Since wikis are a centralized resource on the Web, they support a decentralized group of users that need access to a single knowledge repository. Also, a wiki's content on a Web page can be broken apart or combined with other pages to create the best possible content page. Finally, hyperlinks can create context within the wiki (Wagner, 2004). Content is linked to make connections, but also hyperlinks can indicate relevance. For example, "pages with many links to them indicate a highly useful page" (Wagner, 2004, p. 277). Automatic backlinks, links to the page where the original link originated, can also help create context for the user. All of these functionalities make wikis useful tools for managing knowledge needs. 


\section{Organizational Applications}

Wagner writes that "the combination of ease and speed of publishing content together with the ability of [sic] engage a potentially large group into the knowledge creation process, enables wikis to become a platform for very large and up-to-date knowledge repositories" (2004, p. 283). According to Wagner, wikis are

good tools for addressing ad hoc problems in a distributed knowledge environment where the knowledge being documented frequently changes or would benefit from fast aggregation. Fichter (2005b) also states that wikis are good for collaboration and sharing content, such as codifying best practices and writing documentation. Finally, wikis are successful when used as an online workspace for groups or teams working on a project or when used for collaborative discovery (Fichter, 2005b).

\section{Wikis in Library Reference Services}

\section{Reference Services and Knowledge Management}

According to Gandhi, "reference librarians have long recognized the need to capture, codify, record, and 'fix' the collective knowledge of their colleagues in some sort of explicit knowledge artifact" (2004, p. 374). This recognition stems from the fact that reference librarians have an incredible amount of tacit knowledge regarding library, community, and online resources. No single librarian can know everything, but collectively "reference librarians possess tremendous knowledge about various reference processes and information sources" (Gandhi, 2004, p. 374). Gandhi states that reference librarians need knowledge management systems to capture their communal knowledge. Knowledge management systems are needed in reference services because they can help librarians (a) systematically collect and organize explicit and tacit knowledge, (b) increase efficiency in locating answers to frequently answered questions, (c) improve decisions regarding sources to consult, (d) improve knowledge sharing, (e) acquire more in-depth knowledge of the library and its resources, (f) better understand the types of questions asked at the reference desk, (g) improve collection 
development, and (h) improve patron access to information (Gandhi, 2004). Overall, successful knowledge management in library reference services can help "establish internal benchmarks, identify and record best practices, and create an environment of continuous learning" (Gandhi, 2004, p. 378).

Early attempts to capture the knowledge of reference librarians include handwritten or typed ready reference files and index cards that contained frequently asked questions, typically arranged alphabetically or by subject. Eventually these early systems were computerized to add keyword access to their contents. These automated systems then migrated online, sometimes as static Web pages and other times as more interactive online databases. Again, the advantage to recording the answers to difficult or frequently asked questions is that reference librarians are recording their explicit knowledge about particular sources, as well as tacit knowledge that they have internalized, while creating knowledge artifacts that can be organized, managed, and reused (Gandhi, 2004). Wikis can help reference librarians fill this knowledge management need. In the next three sections, I have outlined some ways wikis can be used in library reference services as a tool to successfully manage knowledge.

Wikis as Internal Knowledge Repositories

As stated before, wikis function extremely well as collaborative knowledge repositories (Fichter, 2005a, 2005b; Frumkin, 2005; Tonkin, 2005; Wagner, 2004, 2005). When used in the context of library reference services, knowledge repositories can take a variety of forms when reference librarians utilize wikis as a knowledge base used within the library. Since wikis can be installed behind firewalls or password protected, they can be set up as an internal, private tool.

Wiki could be used as a ready reference database for frequently asked questions. The advantage to this type of wiki is the ability for anyone easily to add new content at any time or revise content on the fly. Since a wiki is a Web-based tool, it is also readily accessible. Another use for a wiki as a knowledge repository would be as a peer resource guide. For example, subject librarians or librarians 
with other specialties could contribute information and tips on library resources for other librarians. An advantage of having this take place in a wiki is the ease with which librarians can keep the content current. In addition, librarians may discover new tricks or tips they want to share about a resource and it would be very simple for them to add that information to an existing wiki page. In this way, librarians could create new knowledge by building on existing knowledge in the wiki.

Another way to use a wiki as an internal knowledge base would be to organize it around a particular function. For example, many reference departments also provide library instruction, both in academic classrooms and in the library. All of the librarians who teach library instruction classes could contribute to a library instruction wiki specifically tailored for their community of users. This type of wiki could include handouts and tutorials used, teaching techniques and tips, class specific information, and anything else librarians might find useful. An example of a library instruction wiki can be found at the Oregon Library Instruction Wiki (http://instructionwiki.org). This wiki is a resource for anyone, but a similar wiki organized around a specific function like this one could be used within an organization as an internal tool as well.

Wikis as Public Knowledge Repositories

Wikis could also be used as collaborative knowledge repositories for the public in the reference services environment (Cohen, 2005; Frumkin, 2005). The reference librarians at Butler University have created a wiki called "WikiRef" (http://www.seedwiki.com/wiki/butler wikiref) that is intended to be a collaborative review of reference resources available through Butler University Libraries. This wiki is public and open for editing by anyone - not only librarians, faculty, staff, and students - although it was designed primarily as a resource for the Butler University community. An advantage to having an open and public resource like this would be to facilitate a discussion about resources and encourage user participation in the library, in addition to having a resource that features the collective knowledge of its users. Wikis can also be used as subject-specific public 
resource guides. Most libraries publish their subject guides as static Web pages. Providing that information in a wiki open to the public can add more functionality to that type of resource. The "Biz Wiki" (http://www.library.ohiou.edu/subjects/bizwiki) at Ohio University Libraries is an example of a wiki being used in this way. The Biz Wiki (see Figure 2) contains information on the business information resources available through Ohio University Libraries in addition to some business how-to research guides. All of this information was initially written by a reference and instruction librarian, but anyone is free to collaborate, comment, edit, or add new content.

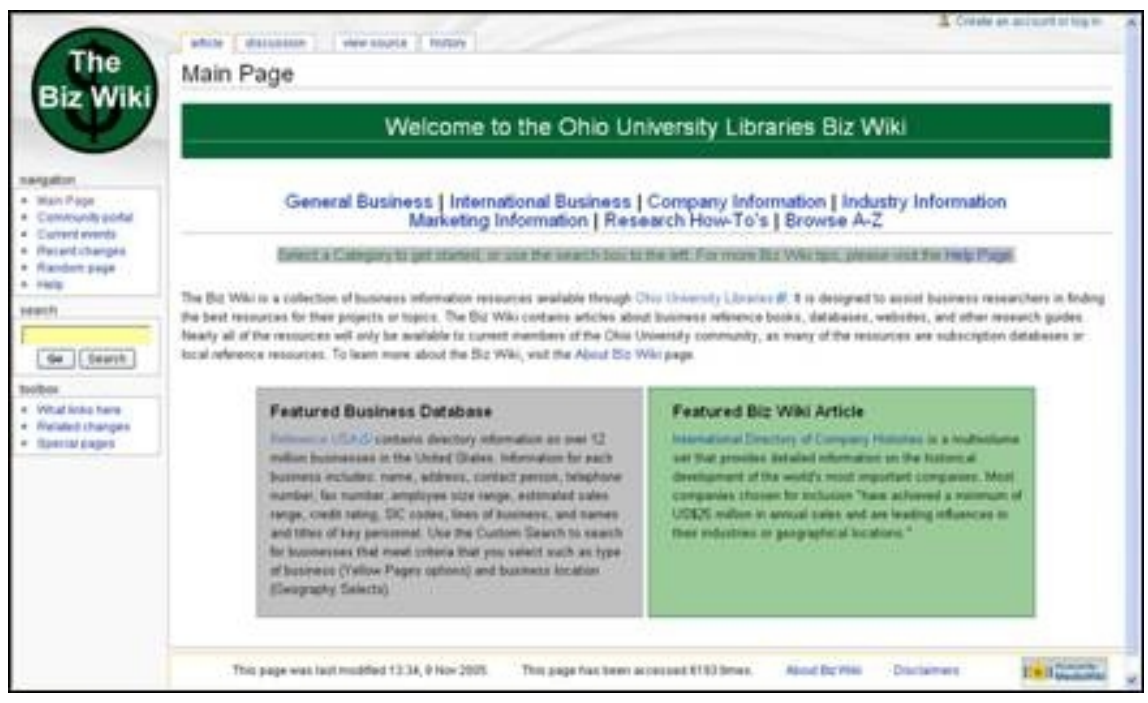

Figure 2. Ohio University Libraries Biz Wiki

\section{Wikis as Collaborative Workspaces}

Finally, wikis can be used as collaborative workspaces to help manage knowledge for specific projects or teams in library reference services (Cohen, 2005; Fichter, 2005a, 2005b; Frumkin, 2005). The wiki could be set up as a private wiki accessible only by a group of team members or it could be set up as publicly readable but editable only by members of the group. The MIT Engineering and Science Libraries B-Team have created a project-specific wiki (http://www.seedwiki.com/wiki/b-team). This team of librarians created the project workspace to investigate the changing information needs of the MIT community 
and make recommendations about the libraries' role within this environment. Librarians could also use a wiki to work on a jointly authored document or to write policies as a group. Although other online tools exist that perform this collaborative writing function (e.g., Writely at http://www.writely.com/ or Writeboard at http://www.writeboard.com/), wikis can be used this way also. Overall, wikis work well as a collaborative workspace and are even more effective when used with distributed work teams.

\section{Conclusion}

There are other ways wikis can be used in library reference services, but this paper has focused on their use in managing knowledge. As a conversational technology, wikis encourage incremental knowledge creation and knowledge sharing, in addition to supporting a decentralized group of users. When wikis are coupled with complementary technologies, they become even more powerful as a knowledge management tool. For example, wikis use RSS to push the recent changes or additions to people who subscribe to a wiki's RSS feed. So, RSS feed readers or aggregators (e.g., Bloglines, Pluck, etc.) are useful tools when used in conjunction with wikis. They help people track changes to a wiki so they know when they want to add content, make additional changes, or comment on new content. All in all, wikis have tremendous value as knowledge management tools in many types of organizations and environments, including library reference services. 


\section{References}

Clyde, L. A. (2005). Wikis. Teacher Librarian, 32(4), 54-56. Retrieved October 25, 2005, from Library Literature \& Information Science database.

Cohen, S. M. (2005). Wiki while you work. Public Libraries, 44(4), 208-209.

Retrieved October 25, 2005, from Library Literature \& Information Science database.

Comparison of wiki software. (2005). In Wikipedia. Retrieved October 25, 2005, from http://en.wikipedia.org/wiki/Comparison of wiki software

Davenport, T. H., \& Prusak, L. (2000). Working knowledge: How organizations manage what they know. Boston: Harvard Business School.

Fichter, D. (2005a, July/August). The many forms of e-collaboration: Blogs, wikis, portals, groupware, discussion boards, and instant messaging. Online, 29(4), 48-50. Retrieved October 25, 2005, from Library Literature \& Information Science database.

Fichter, D. (2005b, September/October). Intranets, wikis, blikis, and collaborative working. Online, 29(5), 47-50. Retrieved October 25, 2005, from Library Literature \& Information Science database.

Frumkin, J. (2005). The wiki and the digital library. OCLC Systems \& Services, 21(1), 18-22. Retrieved October 25, 2005, from Emerald Insight database.

Gandhi, S. (2004). Knowledge management and reference services. The Journal of Academic Librarianship, 30(5), 368-381. Retrieved October 25, 2005, from ScienceDirect database.

Gunnlaugsdottir, J. (2003). Seek and you will find, share and you will benefit: Organising knowledge using groupware systems. International Journal of Information Management, 23(5), 363-380. Retrieved October 25, 2005, from ScienceDirect database.

Jantz, R. (2001). Knowledge management in academic libraries: Special tools and processes to support information professionals. Reference Services Review, 
29(1), 33-39. Retrieved October 25, 2005, from Emerald Insight database.

Leuf, B., \& Cunningham, W. (2001). The wiki way: Quick collaboration on the Web. Boston: Addison-Wesley.

Prusak, L. (2001). Where did knowledge management come from? IBM Systems Journal, 40(4). Retrieved October 25, 2005, from http://www.research.ibm.com/journal/sj/404/prusak.html

Stover, M. (2004). Making tacit knowledge explicit: The ready reference database as codified knowledge. Reference Services Review, 32(2), 164-173. Retrieved October 25, 2005, from Emerald Insight database.

Tonkin, E. (2005, January). Making the case for a wiki. Ariadne, (42). Retrieved October 25, 2005, from http://www.ariadne.ac.uk/issue42/tonkin/

Wagner, C. (2004). Wiki: A technology for conversational knowledge management and group collaboration. Communications of the Association for Information Systems, 13, 265-289. Retrieved October 25, 2005, from Business Source Premier database.

Wagner, C. (2005). Supporting knowledge management in organizations with conversational technologies: Discussion forums, weblogs, and wikis. Journal of Database Management, 16(2), i-viii. Retrieved October 25, 2005, from Business \& Company Resource Center database. 